\title{
Editorial
}

\section{Connected Vehicles: Applications and Communication Challenges}

\author{
Barbara M. Masini, ${ }^{1}$ Gianluigi Ferrari, ${ }^{2}$ Cristiano Silva, ${ }^{3}$ and Ilaria Thibault ${ }^{4}$ \\ ${ }^{1}$ National Research Council (CNR), Institute of Electronics, Computer and Telecommunication Engineering (IEIIT), \\ Wireless Communication Group, Bologna, Italy \\ ${ }^{2}$ Internet of Things (IoT) Lab, Department of Engineering and Architecture, University of Parma, \\ Parma, Italy \\ ${ }^{3}$ Department of Technology, Federal University of São João del-Rei (UFSJ), São João del-Rei, MG, Brazil \\ ${ }^{4}$ Vodafone Group Research and Development, Newbury, UK \\ Correspondence should be addressed to Barbara M. Masini; barbara.masini@ieiit.cnr.it
}

Received 13 July 2017; Accepted 13 July 2017; Published 28 August 2017

Copyright (C) 2017 Barbara M. Masini et al. This is an open access article distributed under the Creative Commons Attribution License, which permits unrestricted use, distribution, and reproduction in any medium, provided the original work is properly cited.

Connected vehicles are expected to be a pillar of a smart society and to revolutionize the way people move. The use of wireless communication technologies integrated on board (or in the driver's or passengers' pockets) is the key to connecting vehicles, infrastructures, and travellers. The widespread use of connected vehicles generates high volumes of transportation-related data, enabling plenty of new applications which, according to Cisco visual networking index, will give a boost to the second fastest growing industry segment (after connected healthcare), with a predicted 37\% compound annual growth rate.

Connected vehicles will improve safety, traffic management, and urban and suburban mobility and will enhance the development of new services like accident prevention, Internet access, Internet backbone, ride-sharing, vehicular social networking, environment monitoring, infotainment, usagebased insurance, preemptive maintenance, and many more. This phenomenon is fostering an acceleration of standardization efforts to define new architectures and requirements for the various emerging vehicular applications scenarios. In addition to well established vehicular communication standards, that is, the IEEE WAVE/802.11p in US and the ETSI ITS G5 in Europe, different working groups within 3GPP have recently completed a set of cellular vehicle-to-anything (V2X) features as part of the LTE-Advanced suite of specifications, and studies on future $5 \mathrm{G}$ systems will take these concepts even further by evaluating how cellular communications together with emerging access technologies-such as visible light and $\mathrm{mm}$-waves-can play a key role in enhancing the on-board intelligence of future vehicles.

This special issue focuses on connected vehicle-driven applications and aims at covering cutting-edge research advances in topics covering standardization, wireless communication technologies challenges, field trials, and tight interworking between on-board vehicle intelligence and wireless communication standards.

A general view on architectures and technologies for connecting vehicles is provided in the following survey by the Guest Editors themselves: "A Survey on InfrastructureBased Vehicular Networks" by C. M. Silva et al. The paper presents an in-depth survey of more than ten years of research on infrastructures, wireless access technologies, and deployment. In fact, even if direct vehicle-to-vehicle communications will allow new challenging applications, the infrastructure still represents the key to let vehicular connectivity available and suitable also nowadays. The paper also identifies the limitations of present technologies and infrastructures and the challenges associated with such infrastructure-based vehicular communications, also highlighting potential solutions. 
Wireless access technologies and ongoing standard solutions are investigated in the following two papers, related to IEEE 802.11p and LTE, respectively.

The paper "An Efficient Channel Access Scheme for Vehicular Ad Hoc Networks" by S. A. Hussain et al. focuses on IEEE 802.11p standard and proposes an efficient channel access scheme for vehicular networks under high vehicular traffic densities and high mobility. In the proposed scheme, the contention window of the random access protocol is dynamically varied according to the instants in correspondence to which vehicles are going to leave the road side unit (RSU) coverage area, giving higher service priority to vehicles leaving sooner the service area. The presented results show that this approach guarantees higher throughput with respect to the standard solution.

The paper "LTE Network Enhancement for Vehicular Safety Communication" by W. Kim and E.-K. Lee focuses on LTE for vehicular applications, as standardized by 3GPP. The authors investigate the feasibility of beacons delivery in terms of network overhead and end-to-end latency, finally proposing three potential solutions to reduce the overhead and the delay, still keeping the safety level high.

Channel modelling is addressed in the following two papers.

The paper "Empirical Study and Modeling of Vehicular Communications at Intersections in the $5 \mathrm{GHz}$ Band" by S. A. Hadiwardoyo et al. presents an empirical study of vehicular communication effectiveness at intersections in the $5 \mathrm{GHz}$ band real field trials at different types of intersections in the city of Valencia (Spain). The goal of this work is to determine the communication restrictions imposed by the different intersections themselves. On the basis of empirical results, the packet delivery probability is also modelled at different distances from the center of the intersection: this allows deriving the expected success ratio when delivering event-based messages.

The paper "Three-Dimensional Vehicle-to-Vehicle Channel Modeling with Multiple Moving Scatterers" by D. Du et al. proposes a generalized 3D channel model with no constraints on the position of local moving scatters. On the basis of this model, the corresponding space-time correlation functions, time correlation functions, and space correlation functions are then analytically investigated for MIMO vehicle-to-vehicle (V2V) links. The theoretical results of the space-Doppler power spectral density are compared with the available measured data showing good agreement and, thus, the applicability and generality of the proposed model.

Safety for pedestrian is considered as example application in the paper "High-Accuracy Tracking Using Ultrawideband Signals for Enhanced Safety of Cyclists" by D. Dardari et al. which focuses on the impact of vehicles on cyclists. After providing a statistical analysis of accidents involving cyclists, the authors present a new risk detection architecture providing high-accuracy localization and tracking of road users based on ultrawideband (UWB) technology. Experimental results show the possibility of achieving centimeter-level localization accuracy and good tracking capabilities, even in harsh propagation environments.
We hope that readers will enjoy the papers published in this special issue and will be inspired for further research work in the topic of connected vehicles.

Barbara M. Masini Gianluigi Ferrari Cristiano Silva Ilaria Thibault 

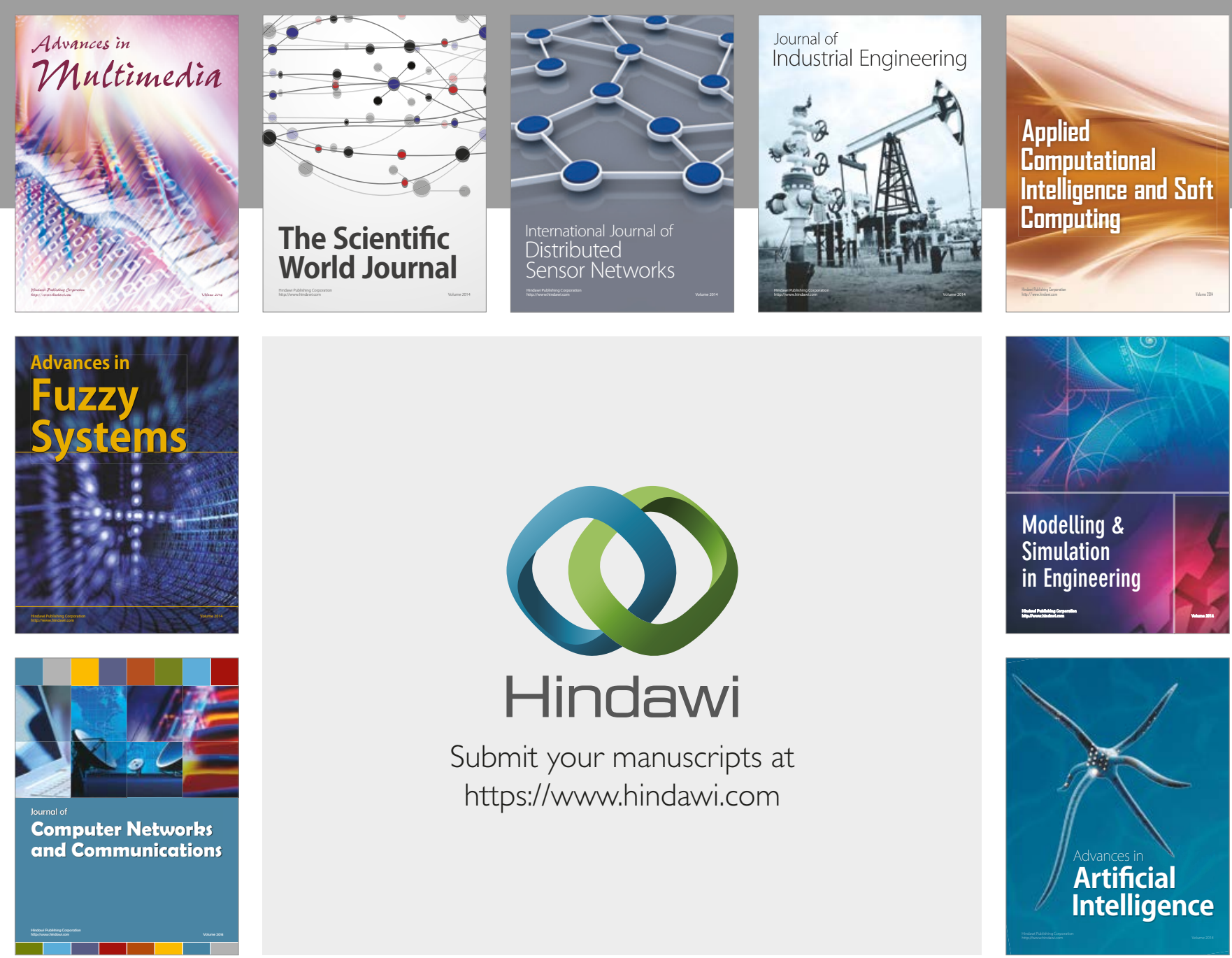

\section{Hindawi}

Submit your manuscripts at

https://www.hindawi.com
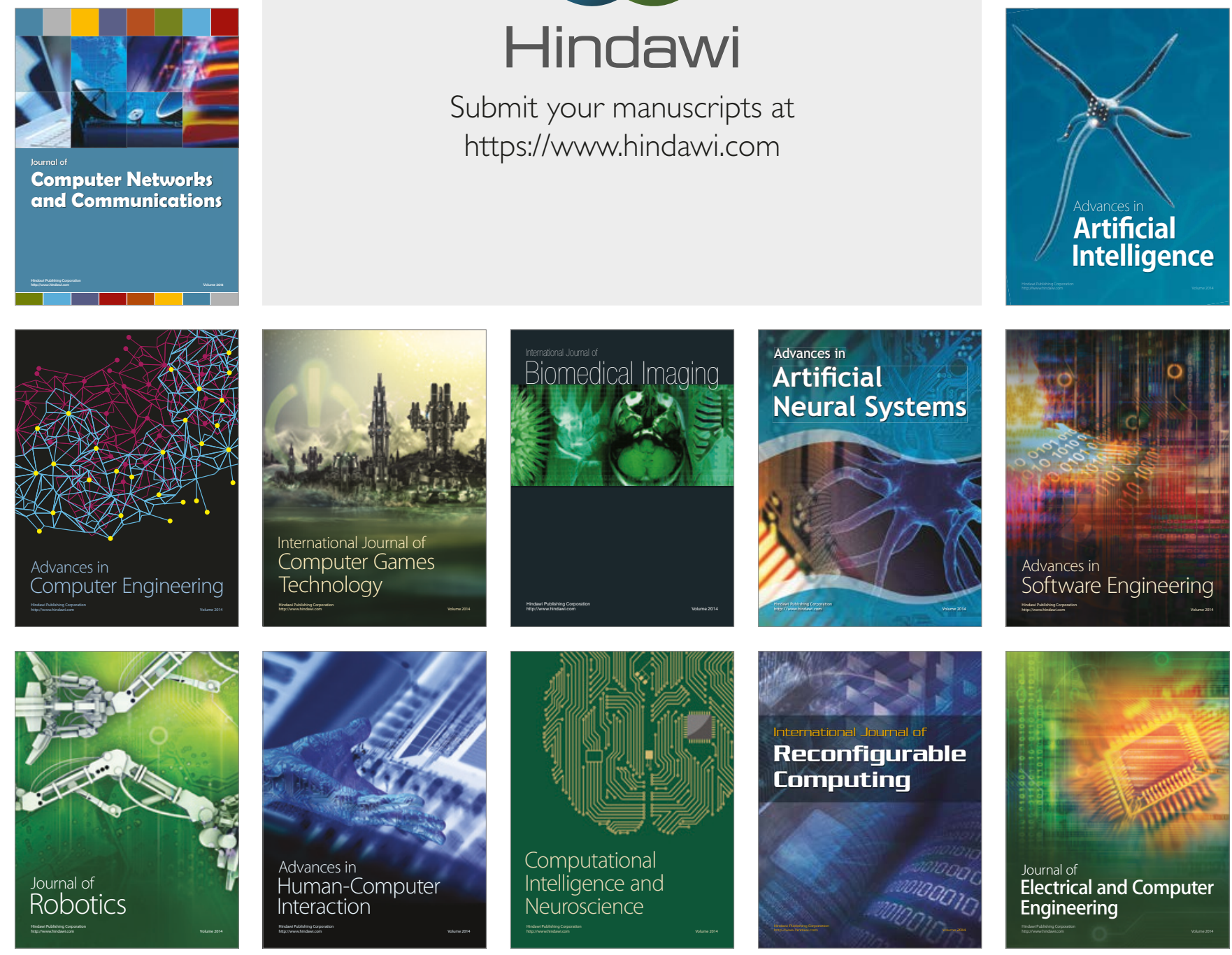